\title{
On the dual frame induced by an invertible frame multiplier
}

\author{
Diana T. Stoeva and Peter Balazs \\ Acoustics Research Institute, Austrian Academy of Sciences, \\ Wohllebengasse 12-14, Vienna A-1040, Austria \\ dstoeva@kfs.oeaw.ac.at, peter.balazs@oeaw.ac.at
}

July 7, 2021

\begin{abstract}
Recently it has been established that given an invertible frame multiplier with semi-normalized symbol, a specific dual of any of the two involved frames can be determined for the inversion purpose. The inverse can be represented as a multiplier with the reciprocal symbol, this particular dual of one of the given frames, and any dual of the other frame. The specific dual is the only one having this property among all Bessel sequences.

In this manuscript we extend the results showing that the specific dual with the above mentioned property is unique among all possible sequences. Furthermore, the symbol is allowed to be not necessarily semi-normalized. Finally we characterize cases when the canonical dual frame and the new specific dual frame coincide.
\end{abstract}

Keywords: Multiplier, Invertibility, Frame, Dual frame, Non-semi-normalized symbol, Non-Bessel sequence

MSC 2000: 42C15, 47A05

\section{Introduction}

For a representation of functions, beside orthonormal bases, other options like Riesz bases [7] or frames [12] exist. Riesz bases extend orthonormal bases giving up with the orthonormality restrictions, but still providing unique representations. Frames extend Riesz bases giving up with the basis-property and allow redundant representations. The redundancy is very desirable in applications, for example in signal processing [10, in particular for noise reduction [8] or signal modification [18]. A natural way to modify functions is first to transform them (via some frame), then multiply the resulting coefficients with a fixed scalar sequence, and then transform back into the original domain via the same or another frame. The operators used to do such a modification are called multipliers. They are of big importance both in mathematics [1, 13, 4] and applications [5, 27, 17, 19. The invertibility of such operators is also of mathematical and practical interest. In this paper we deal with the question on how to represent the inverse as a multiplier involving appropriate dual frames of the given ones. 
Riesz multipliers with semi normalized symbols are always invertible and the inverse can be written as a multiplier with the reciprocal symbol and the canonical dual frames 2. Considering the more general case of frame multipliers with semi-normalized symbols, invertibility is not always the case and even in the case of invertibility, the above mentioned way of inversion does not necessarily work, though it is still possible in certain cases. Investigation of the frame multiplier case was done in [6] where formulas for the inverse were determined involving appropriate dual frames of the given ones. Uniqueness of these dual frames was established among the set of the Bessel sequences. A step further, the SampTA-proceedings [26] was devoted to the investigation of frame multipliers where the symbol is not necessarily semi-normalized and non-Bessel sequences were also involved in the consideration. The results there were announced without proofs. This paper is an extension of [26]. Here we give proofs of the results, as well as provide new results related to characterization of frame multiplier cases where the inversion can be done using precisely the formula from the Riesz multiplier case.

The paper is organized as follows. Section 2 presents the existing and motivating results regarding the particular unique duals involved in representing the inverse multiplier. These results are related to semi-normalized symbols and the assumption of Bessel sequences. The section also points to the main questions, concerning generalizations of the existing results, which are treated in the later sections. In Section 3 the assumption of Bessel sequences is lifted. In Section 4 not necessarily semi-normalized symbols are considered. And finally in Section 5 we comment on and characterize cases when the formula for representing the inverse of a Riesz multiplier, using the canonical duals, hold for redundant frame multipliers.

\section{Basic Definitions, Notation, and Known Facts}

Throughout the paper, $\mathcal{H}$ denotes a separable Hilbert space, $\left(e_{n}\right)_{n=1}^{\infty}-$ an orthonormal basis of $\mathcal{H}, \Phi$ (resp. $\Psi$ ) - a sequence $\left(\phi_{n}\right)_{n=1}^{\infty}\left(\right.$ resp. $\left.\left(\psi_{n}\right)_{n=1}^{\infty}\right)$ with elements from $\mathcal{H}, m$ a complex scalar sequence $\left(m_{n}\right)_{n=1}^{\infty}, \bar{m}$ - the sequence $\left(\overline{m_{n}}\right)_{n=1}^{\infty}$ consisting of the complex conjugates of the elements of $m, 1 / m$ - the sequence $\left(1 / m_{n}\right)_{n=1}^{\infty}, m \Phi$ - the sequence $\left(m_{n} \phi_{n}\right)_{n=1}^{\infty}$.

The sequence $m$ is called semi-normalized if there exist $a, b$ so that $0<a \leq\left|m_{n}\right| \leq$ $b<\infty$ for all $n \in \mathbb{N}$. The term operator is used with the meaning of a linear mapping; an operator $L: \mathcal{H} \rightarrow \mathcal{H}$ is called invertible if it is bounded and bijective.

For extensive presentation on frames we refer to [9, 11, 14, 15]. Recall some needed definitions and facts from frame theory.

The sequence $\Phi$ is called

- a Bessel sequence in $\mathcal{H}$ if there exists $B \in(0, \infty)$ so that $\sum_{n=1}^{\infty}\left|\left\langle f, \phi_{n}\right\rangle\right|^{2} \leq B\|f\|^{2}$ for all $f \in \mathcal{H}$;

- a frame for $\mathcal{H}$ if it is a Bessel sequence in $\mathcal{H}$ and there exists $A \in(0, \infty)$ so that $A\|f\|^{2} \leq \sum_{n=1}^{\infty}\left|\left\langle f, \phi_{n}\right\rangle\right|^{2}$ for all $f \in \mathcal{H}$

- a Riesz basis for $\mathcal{H}$ if it is both a frame for $\mathcal{H}$ and a Schauder basis for $\mathcal{H}$.

Two frames $\Phi$ and $\Psi$ for $\mathcal{H}$ are called equivalent when there exists an invertible operator $L: \mathcal{H} \rightarrow \mathcal{H}$ mapping $\phi_{n}$ to $\psi_{n}, n \in \mathbb{N}$.

A frame $\left(f_{n}\right)_{n=1}^{\infty}$ which satisfies $f=\sum_{n=1}^{\infty}\left\langle f, \phi_{n}\right\rangle f_{n}=\sum_{n=1}^{\infty}\left\langle f, f_{n}\right\rangle \phi_{n}$ for all $f \in \mathcal{H}$ and a given frame $\Phi$ is called a dual frame of $\Phi$. The frame operator of a frame $\Phi$, denoted by $S_{\Phi}$, is determined by $S_{\Phi} f:=\sum_{n=1}^{\infty}\left\langle f, \phi_{n}\right\rangle \phi_{n}$; it is well defined on $\mathcal{H}$ and 
invertible [12. The sequence $\widetilde{\Phi}:=\left(S_{\Phi}^{-1}\left(\phi_{n}\right)\right)_{n=1}^{\infty}$ is a dual frame of $\Phi$ [12, called the canonical dual of $\Phi$.

In addition to dual frames, we also take into consideration sequences which are not necessarily frames and still provide series expansions (examples can be found e.g. in [16, 20, 21, 25]). Given a frame $\Phi$ for $\mathcal{H}$, the sequence $F=\left(f_{n}\right)_{n=1}^{\infty}$ with elements from $\mathcal{H}$ is called

- an analysis pseudo-dual (in short, a-pseudo-dual) of $\Phi$, if $f=\sum_{n=1}^{\infty}\left\langle f, f_{n}\right\rangle \phi_{n}$ for all $f \in \mathcal{H}$.

- a synthesis pseudo-dual (in short, s-pseudo-dual) of $\Phi$, if $f=\sum_{n=1}^{\infty}\left\langle f, \phi_{n}\right\rangle f_{n}$ for all $f \in \mathcal{H}$.

For more on a-pseudo-duals and s-pseudo-duals, see [20.

Given sequences $m, \Phi$, and $\Psi$, the operator $M_{m, \Phi, \Psi}$ determined by

$$
M_{m, \Phi, \Psi} f=\sum_{n=1}^{\infty} m_{n}\left\langle f, \psi_{n}\right\rangle \phi_{n}, f \in \operatorname{dom}\left(M_{m, \Phi, \Psi}\right) \subseteq \mathcal{H}
$$

is called a multiplier and $m$ is called the symbol of $M_{m, \Phi, \Psi}$. The multiplier $M_{m, \Phi, \Psi}$ is called well-defined on $\mathcal{H}$ if its domain is $\mathcal{H}$. When $\Phi$ and $\Psi$ are frames (resp. Riesz bases) for $\mathcal{H}, M_{m, \Phi, \Psi}$ is called a frame multiplier, resp. Riesz multiplier. Frame multipliers with semi-normalized symbols are well-defined on $\mathcal{H}[2$. When $m$ is the constant sequence $(c, c, c, \ldots)$, the multiplier $M_{m, \Phi, \Psi}$ will be denoted by $M_{(c), \Phi, \Psi}$. For works oriented to invertibility of multipliers refer to [6, 22, 24, 25].

Notice that the assumptions of all assertions in the next sections lead to welldefindedness of the multipliers $M_{m, \Phi, \Psi}$ and we will not mention this explicitly in the statements.

\section{Motivation - the case of semi-normalized symbols and Bessel sequences}

Here we review the motivating results which concern multipliers with semi-normalized symbols and take into consideration only Bessel sequences. The starting point for considering the topic was a result about invertibility of multipliers for Riesz bases and representation of the inverse via the canonical duals:

Proposition 2.1 2] Let $\Phi$ and $\Psi$ be Riesz bases for $\mathcal{H}$, and let the symbol $m$ be seminormalized. Then $M_{m, \Phi, \Psi}$ is invertible and

$$
M_{m, \Phi, \Psi}^{-1}=M_{1 / m, \widetilde{\Psi}, \widetilde{\Phi}} .
$$

The above statement has lead to investigation of multipliers for (possibly overcomplete) frames aiming representation of the inverse (in case of invertibility) of a similar type like (10), and has led to the following results:

Theorem 2.2 [6] Let $\Phi$ and $\Psi$ be frames for $\mathcal{H}$, and let the symbol $m$ be semi-normalized. Assume that $M_{m, \Phi, \Psi}$ is invertible. Then the following statements hold.

(a1) There exists a unique dual frame $\Phi^{\dagger}$ of $\Phi$, so that

$$
M_{m, \Phi, \Psi}^{-1}=M_{1 / m, \Psi^{d}, \Phi^{\dagger}}, \forall \text { dual frames } \Psi^{d} \text { of } \Psi .
$$

The frame $\Phi^{\dagger}$ equals the sequence $\left(\left(M_{m, \Phi, \Psi}^{-1}\right)^{*}\left(\overline{m_{n}} \psi_{n}\right)\right)_{n=1}^{\infty}$. 
(b1) There exists a unique dual frame $\Psi^{\dagger}$ of $\Psi$, so that

$$
M_{m, \Phi, \Psi}^{-1}=M_{1 / m, \Psi^{\dagger}, \Phi^{d}}, \forall \text { dual frames } \Phi^{d} \text { of } \Phi .
$$

The frame $\Psi^{\dagger}$ equals the sequence $\left(M_{m, \Phi, \Psi}^{-1}\left(m_{n} \phi_{n}\right)\right)_{n=1}^{\infty}$.

(a2) $\Phi^{\dagger}$ is the only Bessel sequence in $\mathcal{H}$ which satisfies (2).

(b2) $\Psi^{\dagger}$ is the only Bessel sequence in $\mathcal{H}$ which satisfies (3).

(a3) If $F=\left(f_{n}\right)_{n=1}^{\infty}$ is a Bessel sequence in $\mathcal{H}$ which fulfills

$$
M_{m, \Phi, \Psi}^{-1}=M_{1 / m, F, \Phi^{\dagger}}
$$

then $F$ must be a dual frame of $\Psi$.

(b3) If $G=\left(g_{n}\right)_{n=1}^{\infty}$ is a Bessel sequence in $\mathcal{H}$ which fulfills

$$
M_{m, \Phi, \Psi}^{-1}=M_{1 / m, \Psi^{\dagger}, G},
$$

then $G$ must be a dual frame of $\Phi$.

Theorem 2.2 naturally leads to the following questions:

[Q1] Are there non-Bessel sequences, which can be used in the role of $\Phi^{d}$ and $\Psi^{d}$, or in the role of $\Phi^{\dagger}$ and $\Psi^{\dagger}$ ?

[Q2] What about cases when $m$ is not necessarily semi-normalized?

Under the assumptions of Theorem 2.2, the uniquely determined dual frame $\Phi^{\dagger}$ of $\Phi$ (resp. $\Psi^{\dagger}$ of $\Psi$ ) is referred to as the dual frame of $\Phi$ (resp. $\Psi$ ) induced by $M_{m, \Phi, \Psi}$.

While the representations of the inverse in (2) and (3) determine an appropriate analogue of (11) for the general frame case, notice that one may still have precisely the formula (11) even in the overcomplete case (e.g., for every frame $\Phi$ for $\mathcal{H}, M_{(1), \Phi, \Phi}$ is the frame operator of $\Phi$ and so it is invertible and fulfills (1D), but this is certainly not always the case, see [6. Example 4.2]. The statement below presents some sufficient conditions, in certain cases being also necessary, for validity of (10).

Proposition 2.3 6] Let the assumptions of Theorem 2.2 hold. Then

$$
\begin{aligned}
& M_{m, \Phi, \Psi}^{-1}=M_{1 / m, \widetilde{\Psi}, \widetilde{\Phi}} \Leftarrow \Psi \text { is equivalent to } m \Phi \Leftrightarrow \Psi^{\dagger}=\widetilde{\Psi} \\
& M_{m, \Phi, \Psi}^{-1}=M_{1 / m, \widetilde{\Psi}, \widetilde{\Phi}} \Leftarrow \Phi \text { is equivalent to } \bar{m} \Psi \Leftrightarrow \Phi^{\dagger}=\widetilde{\Phi} .
\end{aligned}
$$

For the case $m=(c, c, c, \ldots), c \neq 0$,

$$
\begin{aligned}
M_{(c), \Phi, \Psi}^{-1}=M_{(1 / c), \widetilde{\Psi}, \widetilde{\Phi}} \Leftrightarrow \Psi \text { is equivalent to } \Phi & \Leftrightarrow \quad \Psi^{\dagger}=\widetilde{\Psi} \\
& \Leftrightarrow \quad \Phi^{\dagger}=\widetilde{\Phi} .
\end{aligned}
$$


Notice that the equivalence of the frames $\Psi$ and $m \Phi$ (resp. $\Phi$ and $\bar{m} \Psi$ ) already implies invertibility of $M_{m, \Phi, \Psi}$, and (10) holds. When $\Psi$ is not equivalent to $m \Phi$ and $\Phi$ is not equivalent to $\bar{m} \Psi$, then $M_{m, \Phi, \Psi}$ can still be invertible (see, e.g., [25, Ex. 4.6.3(ii)], where neither $\Psi$ is equivalent to $m \Phi$, nor $\Phi$ is equivalent to $\bar{m} \Psi$, but $M_{m, \Phi, \Psi}$ is invertible; furthermore (11) does not hold) and the question is whether in such cases it may happen at all to have (1):

[Q3] Are the first implications in (4), resp. (5), actually equivalences?

As shown in Proposition 2.3, in the case when $m$ is a constant sequence, the answer of [Q3] is affirmative, so the aim now is to answer the question in the general case of $m$ not necessarily being a constant sequence.

The paper is devoted to the above and related questions. Answers of $[Q 1]$ and $[Q 2]$ were announced in [26] without proofs; they are presented here. In addition, in this paper we also present investigation related to the question [Q3].

\section{On [Q1]: non-Bessel sequences}

In this section we extend Theorem 2.2, taking non-Bessel sequences into consideration, and answer $[Q 1]$, while continuing the numbering from Theorem 2.2

Theorem 3.1 Let the assumptions of Theorem 2.2 hold and let $\Phi^{\dagger}$ and $\Psi^{\dagger}$ be determined by Theorem 2.2. Then the following statements hold.

(a4) $\Phi^{\dagger}$ is the only sequence in $\mathcal{H}$ which satisfies (2).

(b4) $\Psi^{\dagger}$ is the only sequence in $\mathcal{H}$ which satisfies (3).

(a5) $M_{m, \Phi, \Psi}^{-1}=M_{1 / m, \Psi^{s d}, \Phi^{\dagger}}, \forall$ s-pseudo-duals $\Psi^{s d}$ of $\Psi$.

(b5) $M_{m, \Phi, \Psi}^{-1}=M_{1 / m, \Psi^{\dagger}, \Phi^{a d}}, \forall$ a-pseudo-duals $\Phi^{a d}$ of $\Phi$.

(a6) If $F=\left(f_{n}\right)_{n=1}^{\infty}$ is a sequence in $\mathcal{H}$ such that $M_{1 / m, F, \Phi^{\dagger}}$ is well-defined and

$$
M_{m, \Phi, \Psi}^{-1}=M_{1 / m, F, \Phi^{\dagger}}
$$

then $F$ must be an s-pseudo-dual of $\Psi$.

(b6) If $G=\left(g_{n}\right)_{n=1}^{\infty}$ is a sequence in $\mathcal{H}$ such that $M_{1 / m, \Psi^{\dagger}, G}$ is well-defined and

$$
M_{m, \Phi, \Psi}^{-1}=M_{1 / m, \Psi^{\dagger}, G},
$$

then $G$ must be an a-pseudo-dual of $\Phi$.

For the proof of the above proposition, we will use the following statements:

Lemma 3.2 Let $\Phi$ be a frame for $\mathcal{H}$ and let $m$ be such that $m_{n} \neq 0$ for every $n$. Assume that $G=\left(g_{n}\right)_{n=1}^{\infty}$ is a sequence in $\mathcal{H}$ so that $M_{m, G, \Phi^{d}}$ (resp. $\left.M_{m, \Phi^{d}, G}\right)$ is the null-operator on $\mathcal{H}$ for every dual frame $\Phi^{d}$ of $\Phi$. Then $G$ is the null-sequence. 
Proof: Assume that the sequence $G$ is such that $M_{m, G, \Phi^{d}}$ is the null operator on $\mathcal{H}$ for every dual frame $\Phi^{d}=\left(\phi_{n}^{d}\right)_{n=1}^{\infty}$ of $\Phi$. Let $f \in \mathcal{H}$. Then

$$
\sum_{n=1}^{\infty} m_{n}\left\langle f, \phi_{n}^{d}\right\rangle g_{n}=0, \forall \text { dual frame } \Phi^{d} \text { of } \Phi
$$

The dual frames of $\Phi$ are characterized as the sequences $\left(\widetilde{\phi}_{n}+h_{n}-\sum_{j=1}^{\infty}\left\langle\widetilde{\phi}_{n}, \phi_{j}\right\rangle h_{j}\right)_{n=1}^{\infty}$ where $\left(h_{n}\right)_{n=1}^{\infty}$ runs through the Bessel sequences (see, e.g., [11, Theor. 5.6.5]). Using this characterization, we get

$$
\sum_{n=1}^{\infty} m_{n}\left\langle f, \widetilde{\phi}_{n}+h_{n}-\sum_{j=1}^{\infty}\left\langle\widetilde{\phi}_{n}, \phi_{j}\right\rangle h_{j}\right\rangle g_{n}=0, \forall \text { Bessel sequence }\left(h_{n}\right)_{n=1}^{\infty} \text { in } \mathcal{H} .
$$

Using (6) with $\widetilde{\Phi}$ in the role of $\Phi^{d}$, it then follows that

$$
\sum_{n=1}^{\infty} m_{n}\left\langle f, h_{n}-\sum_{j=1}^{\infty}\left\langle\widetilde{\phi}_{n}, \phi_{j}\right\rangle h_{j}\right\rangle g_{n}=0, \forall \text { Bessel sequence }\left(h_{n}\right)_{n=1}^{\infty} \text { in } \mathcal{H} .
$$

Take $\left(h_{n}\right)_{n=1}^{\infty}=\left(e_{1}, 0,0,0, \ldots,\right)$. Then

$$
m_{1}\left\langle f, e_{1}\right\rangle g_{1}-\sum_{n=1}^{\infty} m_{n}\left\langle f,\left\langle\widetilde{\phi}_{n}, \phi_{1}\right\rangle e_{1}\right\rangle g_{n}=0,
$$

implying

$$
m_{1}\left\langle f, e_{1}\right\rangle g_{1}-\left\langle f, e_{1}\right\rangle \sum_{n=1}^{\infty} m_{n}\left\langle\phi_{1}, \widetilde{\phi}_{n}\right\rangle g_{n}=0,
$$

which by (6) leads to $m_{1}\left\langle f, e_{1}\right\rangle g_{1}=0$. Choosing $f=e_{1}$, one comes to the conclusion that $g_{1}=0$.

In a similar way, taking $\left(h_{n}\right)_{n=1}^{\infty}=\left(0,0, \ldots, 0, e_{i}, 0, \ldots\right)$ with $e_{i}$ being at the $i$-th place, $i \geq 2$, we get $g_{i}=0, i \geq 2$.

Assuming that $G$ is a sequence such that $M_{m, \Phi^{d}, G}$ is the null operator on $\mathcal{H}$ for every dual frame $\Phi^{d}=\left(\phi_{n}^{d}\right)_{n=1}^{\infty}$ of $\Phi$, one may proceed with similar techniques to prove that $G$ is the null sequence.

Proof of Theorem 3.1; Throughout the proof, let $M$ denote the multiplier $M_{m, \Phi, \Psi}$.

(a4) Assume that $F=\left(f_{n}\right)_{n=1}^{\infty}$ is a sequence in $\mathcal{H}$ so that $M^{-1}=M_{1 / m, \Psi^{d}, F}$ for every dual frame $\Psi^{d}$ of $\Psi$. By Theorem 2.2 (b1), it follows that $M_{1 / m, \Psi^{\dagger}, \Phi^{d}}=M_{1 / m, F, \Phi^{d}}$ for every dual frame $\Psi^{d}$ of $\Psi$. Then Lemma 3.2 implies that $F=\Psi^{\dagger}$.

(b4) can be proved in a similar way as (a4).

(a5) Assume that $\Psi^{s d}$ is an s-pseudo-dual of $\Psi$. Then for every $f \in \mathcal{H}$, $\sum_{n=1}^{\infty}\left\langle f, \psi_{n}\right\rangle \psi_{n}^{\text {sd }}=f$, which implies that

$$
M^{-1} f=\sum_{n=1}^{\infty}\left\langle M^{-1} f, \psi_{n}\right\rangle \psi_{n}^{s d}=\sum_{n=1}^{\infty} \frac{1}{m_{n}}\left\langle f,\left(M^{-1}\right)^{*}\left(\overline{m_{n}} \psi_{n}\right)\right\rangle \psi_{n}^{s d} .
$$

Since $\Phi^{\dagger}$ is the sequence $\left(\left(M^{-1}\right)^{*}\left(\overline{m_{n}} \psi_{n}\right)\right)_{n=1}^{\infty}$, it now follows that $M_{1 / m, \Psi^{s d}, \Phi^{\dagger}}$ is well defined and equal to $M^{-1}$. 
(b5) Assume that $\Phi^{a d}$ is an a-pseudo-dual of $\Phi$. Then for every $f \in \mathcal{H}$, $\sum_{n=1}^{\infty}\left\langle f, \phi_{n}^{a d}\right\rangle \phi_{n}=f$, which implies that

$$
M^{-1} f=\sum_{n=1}^{\infty} \frac{1}{m_{n}}\left\langle f, \phi_{n}^{a d}\right\rangle M^{-1}\left(m_{n} \phi_{n}\right)
$$

Since $\Psi^{\dagger}$ is the sequence $\left(M^{-1}\left(m_{n} \phi_{n}\right)\right)_{n=1}^{\infty}$, it follows that $M_{1 / m, \Psi^{\dagger}, \Phi^{a d}}$ is well defined and equal to $M^{-1}$.

(a6) Assume that $F=\left(f_{n}\right)_{n=1}^{\infty}$ is a sequence in $\mathcal{H}$ such that $M_{1 / m, F, \Phi^{\dagger}}$ is well-defined and $M^{-1}=M_{1 / m, F, \Phi^{\dagger}}$. Then for every $f \in \mathcal{H}$

$$
M^{-1} f=\sum_{n=1}^{\infty} \frac{1}{m_{n}}\left\langle f, \phi_{n}^{\dagger}\right\rangle f_{n}=\sum_{n=1}^{\infty} \frac{1}{m_{n}}\left\langle f,\left(M^{-1}\right)^{*}\left(\overline{m_{n}} \psi_{n}\right)\right\rangle f_{n}=\sum_{n=1}^{\infty}\left\langle M^{-1} f, \psi_{n}\right\rangle f_{n} .
$$

Therefore, $F$ is an s-pseudo-dual of $\Psi$.

(b6) Assume that $G=\left(g_{n}\right)_{n=1}^{\infty}$ is a sequence in $\mathcal{H}$ such that $M_{1 / m, \Psi^{\dagger}, G}$ is well-defined and $M^{-1}=M_{1 / m, \Psi^{\dagger}, G}$. Then for every $f \in \mathcal{H}$,

$$
f=M M_{1 / m, \Psi^{\dagger}, G} f=M \sum_{n=1}^{\infty} \frac{1}{m_{n}}\left\langle f, g_{n}\right\rangle M^{-1}\left(m_{n} \phi_{n}\right)=\sum_{n=1}^{\infty}\left\langle f, g_{n}\right\rangle \phi_{n},
$$

which implies that $G$ is an a-pseudo-dual of $\Phi$.

\section{On [Q2]: non-semi-normalized symbols}

Here we consider multipliers with symbols not necessarily being semi-normalized, as well as taking into consideration sequences not necessarily being Bessel.

Proposition 4.1 Let $\Phi$ and $\Psi$ be frames for $\mathcal{H}$, and let the symbol $m$ be such that $m_{n} \neq 0$ for every $n$ and the sequence $m \Phi$ be a frame for $\mathcal{H}$. Assume that $M_{m, \Phi, \Psi}$ is invertible. Then the following statements hold.

(i) There exists a unique sequence $\Psi^{\dagger}$ in $\mathcal{H}$ so that

$$
M_{m, \Phi, \Psi}^{-1}=M_{1 / m, \Psi^{\dagger}, \Phi^{a d}}, \forall \text { a-pseudo-duals } \Phi^{a d} \text { of } \Phi,
$$

and it is a dual frame of $\Psi$. Furthermore, $\Psi^{\dagger}=\left(M_{m, \Phi, \Psi}^{-1}\left(m_{n} \phi_{n}\right)\right)_{n=1}^{\infty}$.

(ii) If $G=\left(g_{n}\right)_{n=1}^{\infty}$ is a sequence in $\mathcal{H}$ such that $M_{1 / m, \Psi^{\dagger}, G}$ is well-defined and

$$
M_{m, \Phi, \Psi}^{-1}=M_{1 / m, \Psi^{\dagger}, G}
$$

then $G$ must be an a-pseudo-dual of $\Phi$.

Proof: (i) Consider $M_{m, \Phi, \Psi}=M_{(1), m \Phi, \Psi}$. Then Theorem 2.2 and Theorem 3.1 can be applied to $M_{(1), m \Phi, \Psi}$, implying that there exists a dual frame $\Psi^{\dagger}$ of $\Psi$ so that

$$
M_{(1), m \Phi, \Psi}^{-1}=M_{(1), \Psi^{\dagger},(m \Phi)^{a d}}, \forall \text { a-pseudo-dual }(m \Phi)^{a d} \text { of } m \Phi
$$


and this frame $\Psi^{\dagger}$ is the only sequence in $\mathcal{H}$ satisfying (7); furthermore, $\Psi^{\dagger}=$ $\left(M_{(1), m \Phi, \Psi}^{-1}\left(m_{n} \phi_{n}\right)\right)_{n=1}^{\infty}=\left(M_{m, \Phi, \Psi}^{-1}\left(m_{n} \phi_{n}\right)\right)_{n=1}^{\infty}$. Since the a-pseudo-duals of $m \Phi$ are precisely the sequences $(1 / \bar{m}) \Phi^{a d}$ with $\Phi^{a d}$ being an a-pseudo-dual of $\Phi$, the property (17) is equivalent to

$$
M_{m, \Phi, \Psi}^{-1}=M_{1 / m, \Psi^{\dagger}, \Phi^{a d}}, \forall \text { a-pseudo-duals } \Phi^{a d} \text { of } \Phi .
$$

(ii) Assume that $G=\left(g_{n}\right)_{n=1}^{\infty}$ is a sequence in $\mathcal{H}$ such that $M_{1 / m, \Psi^{\dagger}, G}$ is well-defined and $M_{m, \Phi, \Psi}^{-1}=M_{1 / m, \Psi^{\dagger}, G}$, i.e., $M_{(1), m \Phi, \Psi}^{-1}=M_{(1), \Psi^{\dagger},(1 / m) G}$. By Theorem 3.1 applied to $M_{(1), m \Phi, \Psi}$, we can conclude that $(1 / \bar{m}) G$ is an a-pseudo-duals of $m \Phi$, which implies that $G$ is an a-pseudo-dual of $\Phi$.

Remark: In the above proposition it is assumed that $\Phi$ is a frame for $\mathcal{H}$ and $m$ is such that $m \Phi$ is also a frame for $\mathcal{H}$. Notice that by [23, both $\Phi$ and $m \Phi$ being frames may happen only in cases determined as follows:

- $\Phi$ is a Riesz basis for $\mathcal{H}$ and $m$ is semi-normalized (in this case $m \Phi$ is a Riesz basis for $\mathcal{H})$;

- $\Phi$ is an overcomplete frame for $\mathcal{H}$ and $m \in \ell^{\infty}$, or, $\Phi$ is an overcomplete frame for $\mathcal{H}$ so that no positive constant $a$ fulfills $a<\left\|\phi_{n}\right\|$ for all $n \in \mathbb{N}$ and $m \notin \ell^{\infty}$ (in such cases $m \Phi$ can be an overcomplete frame for $\mathcal{H}$ ).

The above Proposition 4.1 is related to the unique dual frame of $\Psi$ induced by $M_{m, \Phi, \Psi}$. With a similar proof, one can show the validity of the following statement which concerns the unique dual frame of $\Phi$ induced by $M_{m, \Phi, \Psi}$ :

Proposition 4.2 Let $\Phi$ and $\Psi$ be frames for $\mathcal{H}$, and let the symbol $m$ be such that $m_{n} \neq 0$ for every $n$ and the sequence $m \Psi$ is a frame for $\mathcal{H}$. Assume that $M_{m, \Phi, \Psi}$ is invertible. Then the following statements hold.

(i) There exists a unique sequence $\Phi^{\dagger}$ in $\mathcal{H}$ so that

$$
M_{m, \Phi, \Psi}^{-1}=M_{1 / m, \Psi^{s d}, \Phi^{\dagger}}, \forall \text { s-pseudo-duals } \Psi^{s d} \text { of } \Psi,
$$

and it is a dual frame of $\Phi$. Furthermore, $\Phi^{\dagger}=\left(\left(M^{-1}\right)^{*}\left(\overline{m_{n}} \psi_{n}\right)\right)_{n=1}^{\infty}$.

(ii) If $F=\left(f_{n}\right)_{n=1}^{\infty}$ is a sequence in $\mathcal{H}$ such that $M_{1 / m, F, \Phi^{\dagger}}$ is well-defined and

$$
M_{m, \Phi, \Psi}^{-1}=M_{1 / m, F, \Phi^{\dagger}}
$$

then $F$ must be an s-pseudo-dual of $\Psi$.

If the symbol is assumed to be bounded, the assumptions that the weighted sequences is a frame is automatically fulfilled, so in summary:

Corollary 4.3 Let $\Phi$ and $\Psi$ be frames for $\mathcal{H}$, and let the symbol $m$ be such that $m_{n} \neq 0$ for every $n$ and $m \in \ell^{\infty}$. Assume that $M_{m, \Phi, \Psi}$ is invertible. Then Propositions 4.1 and 4.2 apply.

Proof: Write $M_{m, \Phi, \Psi}$ in the way $M_{(1), m \Phi, \Psi}$. Since $m \Phi$ and $\Psi$ are Bessel sequences and $M_{m, \Phi, \Psi}$ is invertible, [22, Prop. 3.1] implies that $m \Phi$ is a frame for $\mathcal{H}$ and thus, Proposition 4.1 applies. In a similar way, writing $M_{m, \Phi, \Psi}=M_{(1), \Phi, \bar{m} \Psi}$ and using [22, Prop. 3.1], it follows that $\bar{m} \Psi$ is a frame for $\mathcal{H}$ and thus, Proposition 4.2 applies.

For an illustration of Corollary 4.3, consider the following example. 
Example 4.4 25] Consider

$$
\begin{aligned}
& \Phi=\left(e_{1}, e_{1},-e_{1}, e_{2}, e_{2},-e_{2}, e_{3}, e_{3},-e_{3}, \ldots\right), \\
& \Psi=\left(e_{1}, e_{1}, e_{1}, e_{2}, \frac{1}{2} e_{2}, \frac{1}{2} e_{2}, e_{3}, \frac{1}{3} e_{3}, \frac{1}{3} e_{3}, \ldots\right), \\
& m=\left(1,1, \quad 1, \quad 1, \quad \frac{1}{2}, \quad \frac{1}{2}, \quad 1, \quad \frac{1}{3}, \quad \frac{1}{3}, \ldots\right) .
\end{aligned}
$$

Then $M_{m, \Phi, \Psi}=I d_{\mathcal{H}}$ and all the assumptions of Corollary 4.3 are satisfied.

To illustrate that Proposition 4.1 covers a larger class of multipliers then Corollary 4.3. consider the following example.

\section{Example 4.5 Consider}

$$
\begin{aligned}
& \Phi=\left(e_{1}, e_{2}, \quad \frac{1}{2} e_{1}, e_{3}, \quad \frac{1}{2^{2}} e_{1}, e_{4}, \quad \frac{1}{2^{3}} e_{1}, e_{5}, \ldots\right), \\
& \Psi=\left(e_{1}, e_{2}, \frac{1}{\sqrt{2}} e_{1}, e_{3}, \frac{1}{\sqrt{2^{2}}} e_{1}, e_{4}, \frac{1}{\sqrt{2^{3}}} e_{1}, e_{5}, \ldots\right), \\
& m=\left(1,1, \quad \sqrt{2}, 1, \quad \sqrt{2^{2}}, 1, \quad \sqrt{2^{3}}, 1, \ldots\right) .
\end{aligned}
$$

Then $M_{m, \Phi, \Psi}=I d_{\mathcal{H}}$ and all the assumptions of Proposition 4.1 are satisfied. Since $m \notin \ell^{\infty}$ and $m \Psi$ is not a frame, Corollary 4.3 and Proposition 4.2 do not apply.

\section{On [Q3]: Inversion using the canonical duals}

Here we show that in general the answer of [Q3] is negative, providing an example:

Example 5.1 Consider the sequences

$$
\begin{aligned}
& \Phi=\left(e_{1}, e_{1},-e_{1}, e_{2}, e_{2},-e_{2}, e_{3}, e_{3},-e_{3}, \ldots\right), \\
& \Psi=\left(e_{1}, e_{1}, e_{1}, e_{2}, e_{2}, e_{2}, e_{3}, e_{3}, e_{3}, \ldots\right), \\
& m=\left(\frac{5+2 \sqrt{5}}{5}, \frac{5-2 \sqrt{5}}{5}, 1, \frac{5+2 \sqrt{5}}{5}, \frac{5-2 \sqrt{5}}{5}, 1, \frac{5+2 \sqrt{5}}{5}, \frac{5-2 \sqrt{5}}{5}, 1, \ldots\right),
\end{aligned}
$$

for which one has that $M_{m, \Phi, \Psi}=I_{\mathcal{H}}$. Here $\widetilde{\Phi}=\frac{1}{3} \Phi, \widetilde{\Psi}=\frac{1}{3} \Psi$, and

$$
M_{1 / m, \widetilde{\Psi}, \widetilde{\Phi}}=\frac{1}{9} M_{1 / m, \Psi, \Phi}=I_{\mathcal{H}}=M_{m, \Phi, \Psi}^{-1} \cdot
$$

However, $\Psi$ is not equivalent to $m \Phi$ and $\Phi$ is not equivalent to $\bar{m} \Psi$.

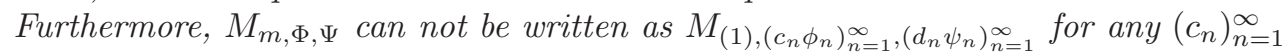
and $\left(d_{n}\right)_{n=1}^{\infty}$ with $\left(c_{n} \phi_{n}\right)_{n=1}^{\infty}$ being equivalent to $\left(d_{n} \psi_{n}\right)_{n=1}^{\infty}$. Assume the opposite, i.e., that $M_{m, \Phi, \Psi}$ can be written as described. Then all $c_{n}$ and $d_{n}$ would be non-zero, because $c_{n} \overline{d_{n}}=m_{n} \neq 0$ for all $n$. Denote $k:=\frac{d_{1}}{c_{1}}$ and let $V: \mathcal{H} \rightarrow \mathcal{H}$ denote the bounded bijective operator mapping every $c_{n} \phi_{n}$ to the corresponding $d_{n} \psi_{n}$. Then $c_{1} V\left(e_{1}\right)=$ $V\left(c_{1} e_{1}\right)=d_{1} e_{1}$ and $-c_{3} V\left(e_{1}\right)=V\left(-c_{3} e_{1}\right)=d_{3} e_{1}$, implying that $d_{3}=-k c_{3}$. Then $0<m_{1}=c_{1} \overline{d_{1}}=\bar{k}\left|c_{1}\right|^{2}$ and $0<m_{3}=c_{3} \overline{d_{3}}=-\bar{k}\left|c_{3}\right|^{2}$ leading to a contradiction.

The above example actually shows that even the weaker question

$$
\text { [Q3'] Under the assumptions of Proposition [2.3, is it valid that }
$$

$$
M_{m, \Phi, \Psi}^{-1}=M_{1 / m, \widetilde{\Psi}, \widetilde{\Phi}} \Leftrightarrow(\Psi \text { is equivalent to } m \Phi) \text { or }(\Phi \text { is equivalent to } \bar{m} \Psi) \text { ? }
$$

has a negative answer in general.

As shown in Proposition 2.3 for the class of frame multipliers with constant symbols, the answer of $[Q 3]$ is affirmative. In the following we determine a larger class of multipliers, where the answer of $[Q 3]$ is still affirmative. When a frame $\Phi$ is multiplied with a 
weight $m$, and the result $m \Phi$ is a frame, i.e. a weighted frame [3], the conjugate reciprocal weighted canonical dual frame $\frac{1}{\bar{m} \widetilde{\Phi}}$ is always a dual frame of $m \Phi$. If $\frac{1}{\bar{m} \widetilde{\Phi}}$ is precisely the canonical dual of $m \Phi$, then the answer of [Q3] is affirmative and this in particular extends the formula (10) for Riesz multipliers to a certain class of frame multipliers:

Proposition 5.2 Let a frame $\Phi$ for $\mathcal{H}$ and a sequence $m$ with $m_{n} \neq 0$ for all $n$ be such that $m \Phi$ is a frame for $\mathcal{H}$ with the property $\widetilde{m \Phi}=\frac{1}{\bar{m}} \widetilde{\Phi}$. Then for any frame $\Psi$ for $\mathcal{H}$ :

$$
\begin{aligned}
M_{m, \Phi, \Psi} \text { is invertible and (1) holds } & \Leftrightarrow \Psi \text { is equivalent to } m \Phi \\
& \Leftrightarrow M_{m, \Phi, \Psi} \text { is invertible and } \Psi^{\dagger}=\widetilde{\Psi} .
\end{aligned}
$$

Proof: By [6, Theor. 4.6], which concerns frame multipliers with constant symbols, we have that

$$
\begin{aligned}
\Psi \text { is equivalent to } m \Phi & \Leftrightarrow M_{(1), m \Phi, \Psi} \text { is invertible and } M_{1, m \Phi, \Psi}^{-1}=M_{(1), \widetilde{\Psi}, \widetilde{\Phi}} \\
& \Leftrightarrow M_{(1), m \Phi, \Psi} \text { is invertible and }\left(M_{(1), m \Phi, \Psi}^{-1}\left(m_{n} \phi_{n}\right)\right)_{n=1}^{\infty}=\widetilde{\Psi} .
\end{aligned}
$$

Using the assumption $\widetilde{m \Phi}=\frac{1}{m} \widetilde{\Phi}$, it follows that $M_{(1), \widetilde{\Psi}, \widetilde{m \Phi}}$ is actually the multiplier $M_{1 / m, \widetilde{\Psi}, \widetilde{\Phi}}$ and this completes the proof of the first equivalence. For the second equivalence, use Proposition 4.1(i).

In a similar way as in Proposition 5.2, one can show validity of the following statement:

Proposition 5.3 Let a frame $\Psi$ for $\mathcal{H}$ and a sequence $m$ with $m_{n} \neq 0$ for all $n$ be such that $\bar{m} \Psi$ is a frame for $\mathcal{H}$ with the property $\widetilde{m} \Psi=\frac{1}{m} \widetilde{\Psi}$. Then for any frame $\Phi$ for $\mathcal{H}$ :

$$
\begin{aligned}
M_{m, \Phi, \Psi} \text { is invertible and (1) holds } & \Leftrightarrow \Phi \text { is equivalent to } \bar{m} \Psi \\
& \Leftrightarrow M_{m, \Phi, \Psi} \text { is invertible and } \Phi^{\dagger}=\widetilde{\Phi} .
\end{aligned}
$$

Finally we can state a result for symbols with constant amplitude but varying phase:

Corollary 5.4 Let $\Phi$ and $\Psi$ be frames for $\mathcal{H}$ and let $m$ be such that $\left|m_{n}\right|=c \neq 0$ for every $n$. Then

$$
\begin{aligned}
M_{m, \Phi, \Psi} \text { is invertible and (1) holds } & \Leftrightarrow \Psi \text { is equivalent to } m \Phi \\
& \Leftrightarrow \Phi \text { is equivalent to } \bar{m} \Psi .
\end{aligned}
$$

Proof: Clearly, $m \Phi$ and $\bar{m} \Psi$ are frames for $\mathcal{H}$. Furthermore, $S_{m \Phi}=c^{2} S_{\Phi}$, implying that

$$
S_{m \Phi}^{-1}\left(m_{n} \phi_{n}\right)=\frac{m_{n}}{c^{2}} S_{\Phi}^{-1}\left(\phi_{n}\right)=\frac{1}{\overline{m_{n}}} S_{\Phi}^{-1}\left(\phi_{n}\right) .
$$

Therefore, $\widetilde{m \Phi}=\frac{1}{m} \widetilde{\Phi}$. In a similar way, it follows that $\widetilde{m \Psi}=\frac{1}{m} \widetilde{\Psi}$. Now Propositions 5.2 and 5.3 complete the proof.

As a simple example illustrating Corollary [5.4 consider the frames $\Phi=$ $\left(e_{1}, e_{1}, e_{2}, e_{2}, e_{3}, e_{3}, \ldots\right)$ and $\Psi=\left(e_{1},-e_{1}, e_{2},-e_{2}, e_{3},-e_{3}, \ldots\right)$, and the sequence $m=$ $(1,-1,1,-1,1,-1, \ldots)$, for which one has $\Psi=m \Phi$ and $\Phi=\bar{m} \Psi$.

ACKNOWLEDGEMENT: The authors acknowledge support from the Austrian Science Fund (FWF) START-project FLAME ('Frames and Linear Operators for Acoustical Modeling and Parameter Estimation'; Y 551-N13). 
ON THE DUAL FRAME INDUCED BY AN INVERTIBLE FRAME MULTIPLIER

\section{References}

[1] M. Arias and M. Pacheco. Bessel fusion multipliers. J. Math. Anal. Appl., 348(2):581-588, 2008.

[2] P. Balazs. Basic definition and properties of Bessel multipliers. J. Math. Anal. Appl., 325(1):571-585, 2007.

[3] P. Balazs, J.-P. Antoine, and A. Grybos. Weighted and controlled frames: Mutual relationship and first numerical properties. Int. J. Wavelets Multiresolut. Inf. Process., 8(1):109-132, 2010.

[4] P. Balazs, D. Bayer, and A. Rahimi. Multipliers for continuous frames in Hilbert spaces. J. Phys. A: Math. Theor., 45(24):244023, 2012.

[5] P. Balazs, B. Laback, G. Eckel, and W. Deutsch. Time-frequency sparsity by removing perceptually irrelevant components using a simple model of simultaneous masking. IEEE Transactions on Audio, Speech, and Language Processing, 18(1):3449, 2010.

[6] P. Balazs and D. T. Stoeva. Representation of the inverse of a frame multiplier. J. Math. Anal. Appl., 422(2):981-994, 2015.

[7] N. K. Bari. Biorthogonal systems and bases in Hilbert space. Uch. Zap. Mosk. Gos. Univ., 148:69-107, 1951.

[8] H. Bölcskei and F. Hlawatsch. Noise reduction in oversampled filter banks using predictive quantization. IEEE Transactions on Information Theory, 47:155-172, 2001.

[9] P. G. Casazza. The art of frame theory. Taiwanese J. Math., 4(2):129-201, 2000.

[10] L. Chai, J. Zhang, C. Zhang, and E. Mosca. Bound ratio minimization of filter bank frames. Signal Processing, IEEE Transactions on, 58(1):209 -220, 2010.

[11] O. Christensen. An Introduction to Frames and Riesz Bases. Applied and Numerical Harmonic Analysis. Birkhäuser, Boston, 2003.

[12] R. Duffin and A. Schaeffer. A class of nonharmonic Fourier series. Trans. Am. Math. Soc., 72:341-366, 1952.

[13] H. G. Feichtinger and K. Nowak. A first survey of Gabor multipliers. Feichtinger, Hans G. (ed.) et al., Advances in Gabor analysis. Basel: Birkhäuser. Applied and Numerical Harmonic Analysis. 99-128 (2003)., 2003.

[14] K. Gröchenig. Foundations of Time-Frequency Analysis. Applied and Numerical Harmonic Analysis. Birkhäuser, Boston, 2001.

[15] C. Heil. A Basis Theory Primer. Expanded ed. Applied and Numerical Harmonic Analysis. Birkhäuser, Basel, 2011.

[16] S. Li and H. Ogawa. Pseudo-duals of frames with applications. Appl. Comput. Harmon. Anal., 11(2):289-304, 2001. 
[17] P. Majdak, P. Balazs, W. Kreuzer, and M. Dörfler. A time-frequency method for increasing the signal-to-noise ratio in system identification with exponential sweeps. In Proceedings of the 36th International Conference on Acoustics, Speech and Signal Processing, ICASSP 2011, Prag, pages 3812 -3815. IEEE, 2011.

[18] G. Matz and F. Hlawatsch. Time-frequency transfer function calculus of linear timevarying systems, chapter 4.7 in 'Time-Frequency Signal Analysis and Processing: A Comprehensive Reference', ed. B. Boashas, pages 135-144. Oxford (UK): Elsevier, 2003.

[19] A. Olivero, B. Torresani, and R. Kronland-Martinet. A class of algorithms for time-frequency multiplier estimation. IEEE Transactions on Audio, Speech, and Language Processing, 21(8):1550-1559, 2013.

[20] D. T. Stoeva. Characterization of frames in Banach spaces and application to Hilbert frames. preprint.

[21] D. T. Stoeva. $X_{d}$-frames in Banach spaces and their duals. Int. J. Pure Appl. Math., 52(1):1-14, 2009.

[22] D. T. Stoeva and P. Balazs. Invertibility of multipliers. Appl. Comput. Harmon. Anal., 33(2):292-299, 2012.

[23] D. T. Stoeva and P. Balazs. Weighted frames and frame multipliers. Annual of the University of Architecture, Civil Engineering and Geodesy, XLIII-XLIV 2004-2009 (Fasc. II Mathematics Mechanics):33-42, 2012.

[24] D. T. Stoeva and P. Balazs. Canonical forms of unconditionally convergent multipliers. J. Math. Anal. Appl., 399(1):252-259, 2013.

[25] D. T. Stoeva and P. Balazs. Detailed characterization of conditions for the unconditional convergence and invertibility of multipliers. Sampl. Theory Signal Image Process., 12(2-3):87-125, 2013.

[26] D. T. Stoeva and P. Balazs. The dual frame induced by an invertible frame multiplier. In Sampling Theory and Applications (SampTA), 2015 International Conference on, pages 101-104. IEEE, 2015.

[27] D. Wang and G. J. Brown. Computational Auditory Scene Analysis: Principles, Algorithms, and Applications. Wiley-IEEE Press, 2006. 